UCRL-JC-122972

PREPRINT

CONF $-951155--43$

\title{
A Molecular Dynamics Simulation Study of Defect Production in Vanadium
}

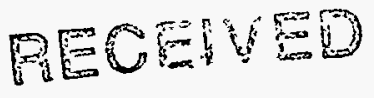

PER 066 TSE

K. Morishita

T.:Diaz de la Rubia
OSTI

This paper was prepared for submittal to the

Materials Research Society 1995 Fall Meeting

Boston, MA

November 27-December 2, 1995

January 23, 1995

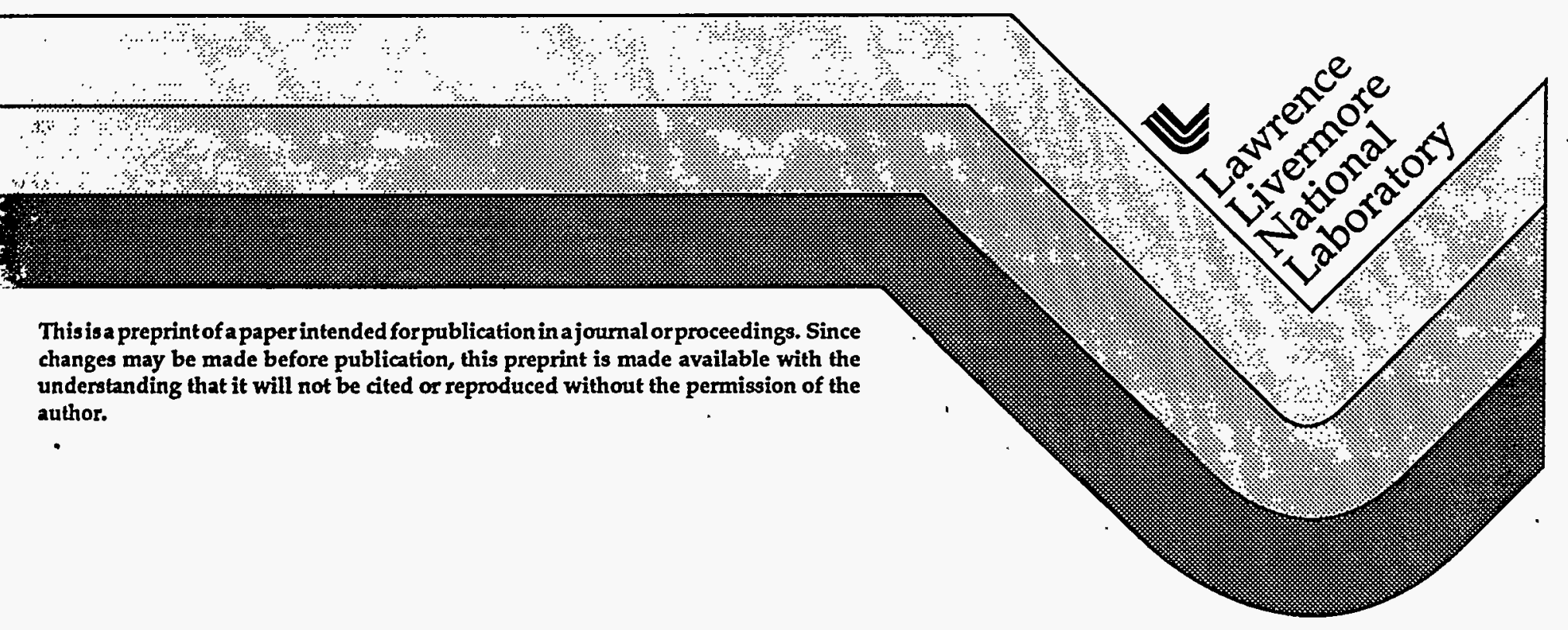




\section{DISCLAIMER}

This document was prepared as an account of work sponsored by an agency of the United States Government. Neither the United States Government nor the University of California nor any of their employees, makes any warranty, express or implied, or assumes any legal liability or responsibility for the accuracy, completeness, or osefulness of any information, apparatus, product, or process disclosed, or represents that its use would not infringe privately owned rights. Reference herein to any specificcommercial - products, process, or service by trade name, trademark, manufacturer, or otherwise, does not necessarily constitute or imply its endorsement, recommendation, or favoring by the United States Government or the University of California. The views and opinions of authors expressed herein do not necessarily state or reflect those of the United States Government or the University of California, and shall not be used for advertising or product endorsement purposes. 


\title{
A MOLECULAR DYNAMICS SIMULATION STUDY OF DEFECT PRODUCTION IN VANADIUM
}

\author{
K. MORISHITA ${ }^{1,2}$ and T. DIAZ DE LA RUBIA ${ }^{1}$
}

${ }^{1}$ Lawrence Livermore National Laboratory, P.O. Box 808, L-268, Livermore, CA 94550.

2 University of Tokyo, Department of Quantum Engineering and Systems Science, 7-3-1 Hongo, Bunkyo-ku, Tokyo 113, Japan

\begin{abstract}
We performed molecular dynamics simulations to investigate the process of defect production in pure vanadium. The interaction of atoms was described by the EAM interatomic potential modified at short range to merge smoothly with the universal potential for description of the high energy recoils in cascades. The melting point of this EAM model of vanadium was found to be consistent with the experimental melting temperature. The threshold energies of displacement events in the model system are also consistent with experimental minimum threshold in vanadium, and its average was found to be $44 \mathrm{eV}$. We evaluated the efficiencies of defect production in the displacement events initiated by recoils with kinetic energy up to $5 \mathrm{keV}$, and found that the probability of cluster formation is smaller than that of simulated events in fcc metals reported in the literature.
\end{abstract}

\section{INTRODUCTION}

Microstructural evolution during irradiation is a complicated, non-linear function of the initial defect production. The changes in mechanical properties due to irradiation are commonly supposed to result from irradiation-induced microstructural changes, such as the formation of point defects, dislocation loops, voids, surface craters and solute segregation. It is, therefore, critical that a clear understanding of these radiation damage processes be achieved, not only for prediction of a given material's response to irradiation but also for development of new, advanced materials. A physical description of the radiation damage process involves varying time- and length-scales from ballistic binary collisions to collective atomic motion in the thermal spike stage followed by the thermal activation process. The time scale of the radiation effects relevant to the field of nuclear materials ranges widely from $10^{-15}$ to $10^{7}$ seconds, and the length scale ranges, also widely, from $10^{-9}$ to $10^{-3}$ meters.

The displacement cascade [1] is of fundamental importance to microstructural changes since it plays a role as a production source of defects in irradiated materials. Moreover, debris of pointand clustered-defects produced by the cascade has recently been a subject of increased interest since it has been suggested that this debris induces a diffusion bias [2] which acts during subsequent microstructural evolution. Molecular dynamics $(\mathrm{MD})$ simulations can be used to study the space and time development of the cascade process [3-8]. With our MDCASK code [9], we can study systems with up to $10^{6}$ atoms on massively parallel computers.

Vanadium-based alloys have significant advantages for use as structural materials in nuclear fusion devices because of their low activation and high radiation resistance. In the present study, in order to provide a first step towards the comprehensive model simulation of radiation effects in these alloys, we investigate cascade dynamics in pure vanadium at the atomic level by $\mathrm{MD}$ simulations with a many-body interatomic potential. First, we evaluated the displacement threshold energies and melting temperature of the model potential system. Second, we simulate the cascade process in vanadium for primary recoils with energy up to $5 \mathrm{keV}$. We discuss the production efficiencies of point- and clustered-defects produced by cascades in vanadium. 
Table 1 Comparison of EAM-type many body potentials for vanadium. The activation energies of vacancy formation, $E_{v}$, vacancy migration, $E_{v}{ }^{m}$ and self diffusion, $Q_{v}$, and the fcc-bcc energy difference are listed in unit of electron volts.

\begin{tabular}{l|lllll}
\hline & $\mathrm{E}_{\mathrm{v}}^{\mathrm{f}}$ & $\mathrm{E}_{\mathrm{v}}^{\mathrm{m}}$ & $\mathrm{Q}_{\mathrm{v}}$ & $\mathrm{E}_{\mathrm{fcc}}-\mathrm{E}_{\mathrm{bcc}}$ & Ref. \\
\hline & & & & & \\
Adams \& Foiles & 2.22 & 0.98 & 3.20 & 0.035 & {$[10]$} \\
Johnson \& Oh & 2.02 & 0.71 & 2.73 & 0.19 & {$[11]$} \\
Finnis \& Sinclair & 1.83 & 0.72 & 2.55 & 0.14 & {$[12]$} \\
Ouyang \& Zhang & & 0.76 & 2.58 & & {$[13]$} \\
Wang \& Boercker & 1.58 & & & 0.046 & {$[14]$} \\
\hline experiment & 2.1 & 0.5 & 2.9 & 0.17 & {$[15]$} \\
\hline ab-initio LMTO & & & & 0.30 & $[1]]$ \\
\hline \hline
\end{tabular}

\section{MOLECULAR DYNAMICS MODEL}

All the simulations in the present study were performed with the molecular dynamics code, MDCASK [9] which runs efficiently on the Cray-2 supercomputer and T3D massively parallel computer at Lawrence Livermore National Laboratory. In order to evaluate existing interatomic potentials for vanadium, we compared the defect properties and fcc-bcc energy difference of several published potentials [10-15] to the experimental data [16] and ab-initio LMTO calculation results [17], respectively, as shown in Table 1. Based on the results, we use the Johnson and Oh EAM-potential [11] for our simulations. Since this EAM potential was, however, developed by fitting to just near equilibrium behavior of vanadium, we modified the high energy part of the potential for description of high energy recoils in cascades. The potential was splined to the universal potential of Biersack and Ziegler (ZBL) [18] by a similar scheme to that of Proennecke et al [19].

\section{RESULTS AND DISCUSSION}

\section{VALIDATION OF INTERATOMICPOTENTIAL}

To validate our model potential (EAM-ZBL potential) for cascade dynamics simulation, the displacement threshold energies and melting temperature were investigated. First, we evaluated displacement threshold energies for the various directions of recoil atom. The initial lattice temperature was $0 \mathrm{~K}$. The threshold energies of our model potential are shown in Fig. 1. The threshold energy for displacing an atom is highly dependent on the direction of recoil atoms, as many previous theoretical and experimental studies have shown [20-22]. The stable configuration of the produced interstitial is found to be the split configuration whose axis lies along $\langle 110\rangle$, which is generally the most stable configuration of interstitials in bcc metals. Our result shows a minimum threshold of $\sim 25 \mathrm{eV}$, which is consistent with the experimental minimum threshold [23]. An average value over all directions is found to be $44 \mathrm{eV}$, in excellent agreement with the suggested ASTM standard [24].

Second, we evaluated the melting temperature of our model potential system. Ari MD crystal containing a stacking fault in the center was annealed at several temperatures ranging from 2100 to $2400 \mathrm{~K}$ during several psec. No melting was observed at $2100 \mathrm{~K}$. However, melting was observed to nucleate at the defect layer during $4 \mathrm{psec}$ simulation at more than $2200 \mathrm{~K}$. To estimate crystalline order upon melting quantitatively, we investigated the squared magnitude of the planar 


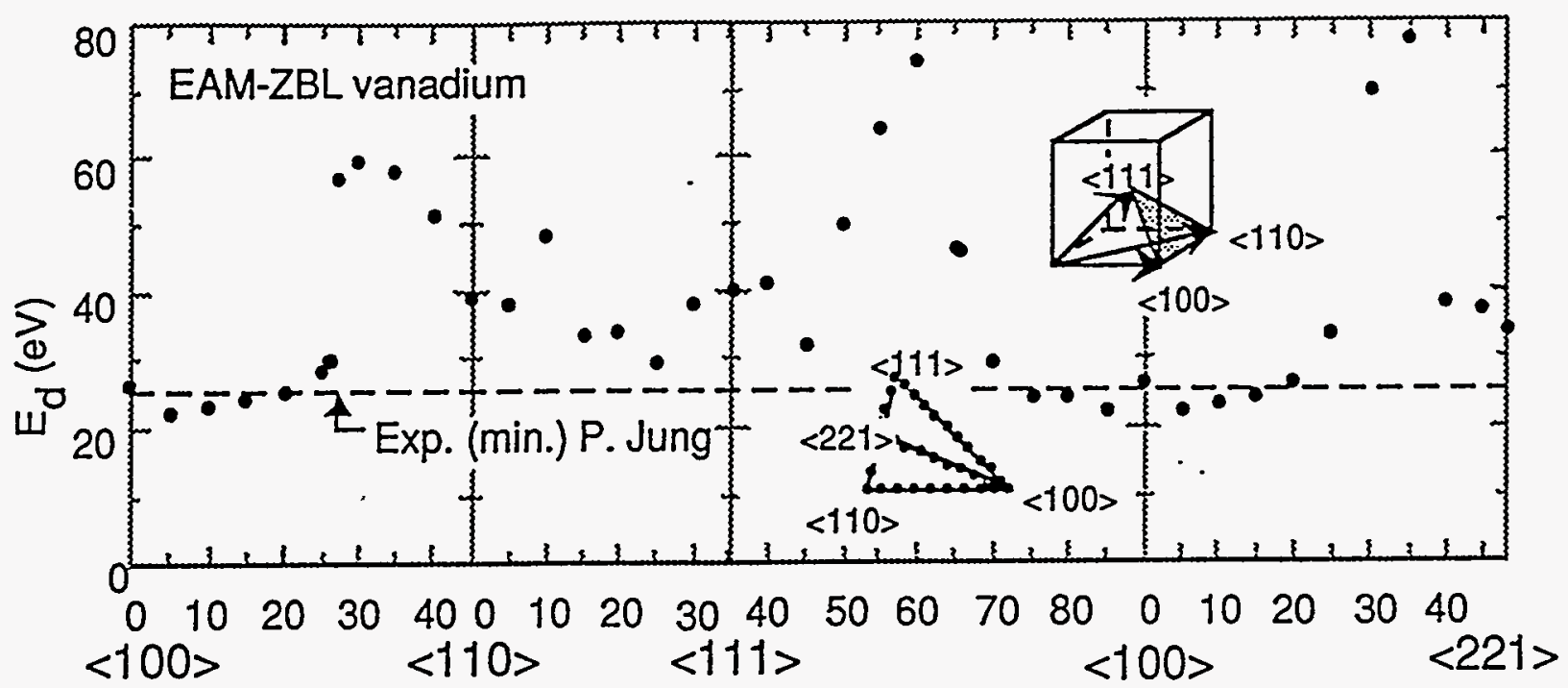

Fig. 1 Displacement threshold energies for point defect production in vanadium. Final configuration of displaced atoms is observed to be $\langle 110\rangle$ split, the most stable configuration of an interstitial in bcc metals.

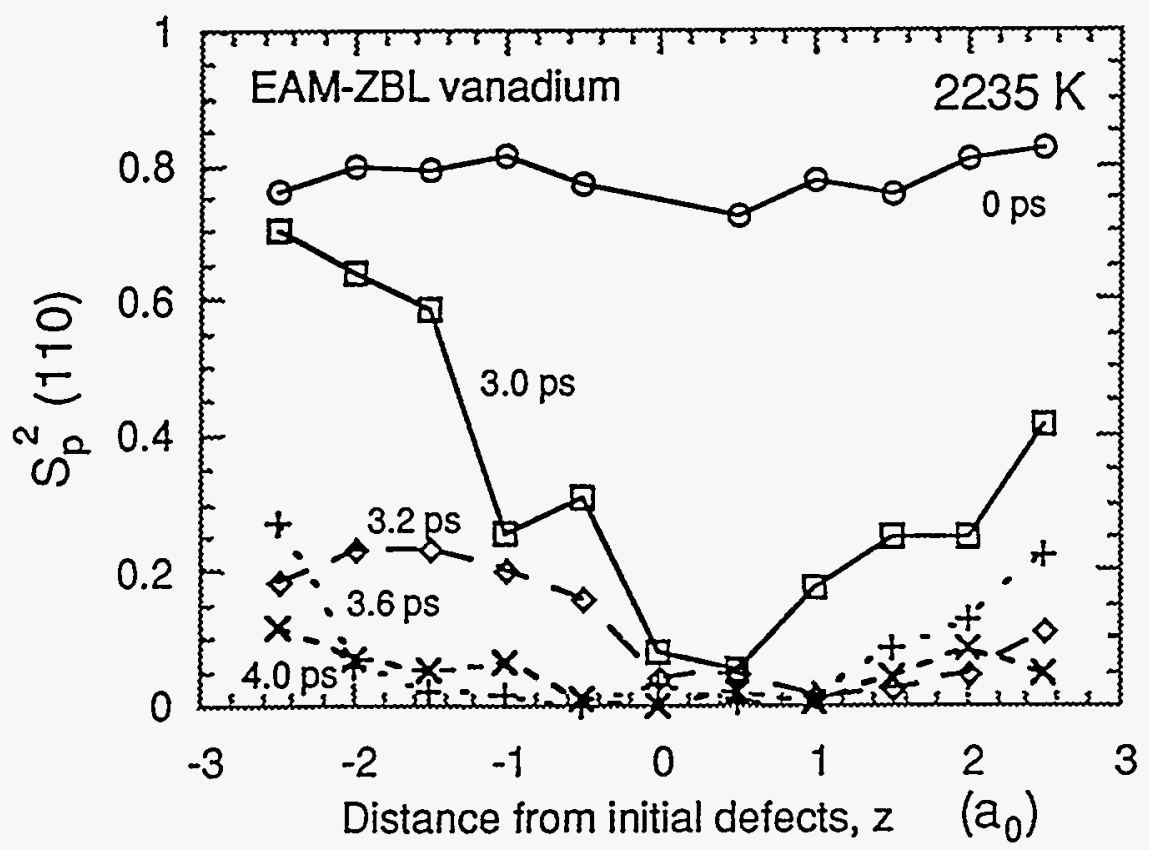

Fig. 2 Propagation of defect-nucleated melting in vanadium at $2235 \mathrm{~K}$. Initial defects are located at $z=0$ at the beginning. The melting is characterized by planar structure factor of $k=(110)$. 


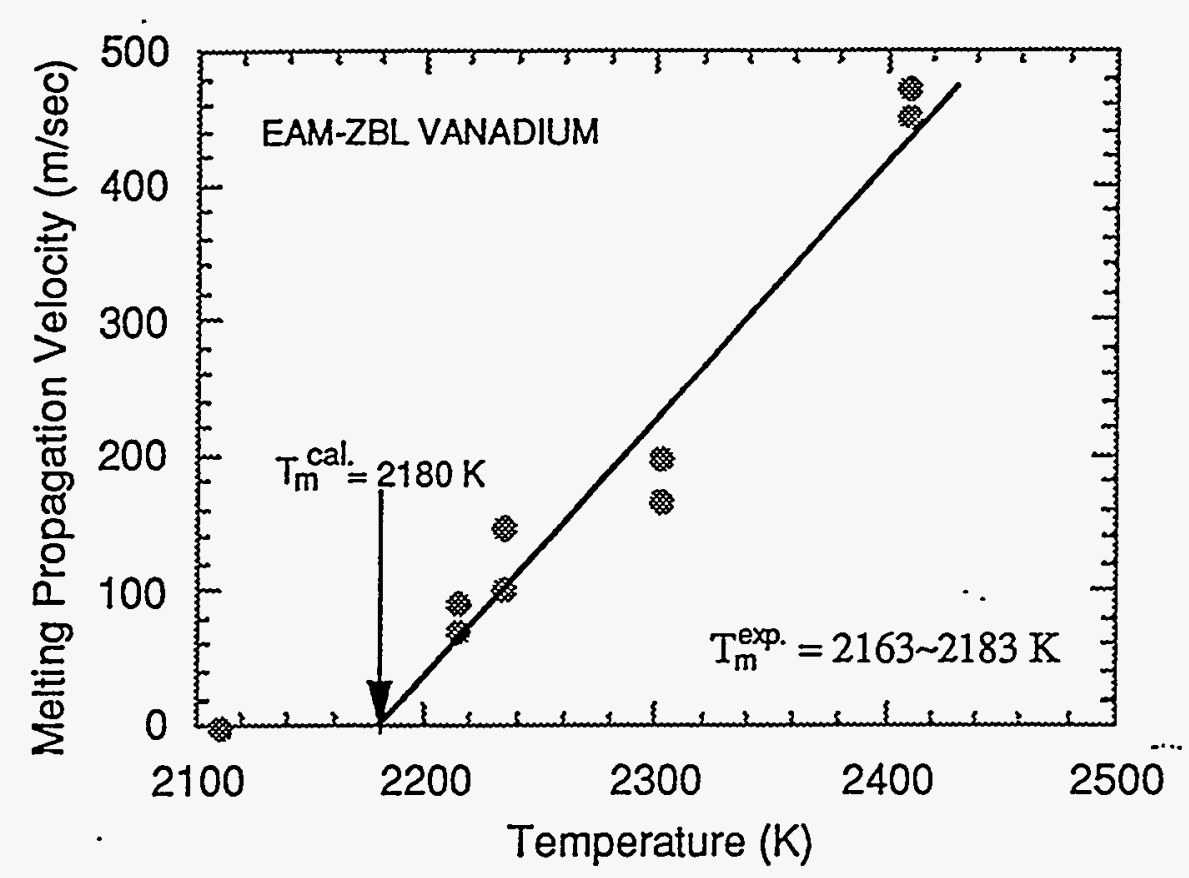

Fig. 3 The propagation velocity of defect nucleated melting as a function of lattice temperature. The calculated melting temperature, $2180 \mathrm{~K}$, is consistent with experimental value.

structure factor, $\mathrm{S}_{\mathrm{p}}^{2}(\mathrm{k})$, as defined by

$$
\mathrm{S}_{\mathrm{p}}^{2}(k)=\left[\frac{1}{\mathrm{~N}} \sum_{\mathrm{i}=1}^{\mathrm{N}} \cos \left(k \bullet q_{\mathrm{i}}\right)\right]^{2}+\left[\frac{1}{\mathrm{~N}} \sum_{\mathrm{i}=1}^{\mathrm{N}} \sin \left(k \bullet q_{\mathrm{i}}\right)\right]^{2},
$$

where $k$ is a reciprocal lattice vector and $q_{\mathrm{i}}$ is the position vector of $i$-th atom in a given plane. For an ideal crystal lattice at zero temperature, $\mathrm{S}_{\mathrm{p}}{ }^{2}(\mathrm{k})$ equals unity for any wave vector $k$, and in the liquid state it fluctuates near zero. Fig. 2 shows the values of the planar structure factor for $(001)$ planes in the MD crystal heated at $2235 \mathrm{~K}$. As shown in the figure, atomic disordering due to melting is observed to take place from the central $(001)$ plane, $z=0.0$ where the defects were initially introduced. The propagation velocity of the melting front depends on the lattice temperature, and goes to zero at the melting point. Fig. 3 shows the melt propagation velocity as a function of temperature. An extrapolation to zero velocity yields a melting temperature of $2180 \mathrm{~K}$, which is in agreement with experimental melting point (2163-2183 K) [25].

\section{DEFECT PRODUCTION}

MD simulations of displacement cascade in vanadium were performed using the EAM-ZBL potential at initial lattice temperature of $10 \mathrm{~K}$. In the present study, primary recoil atoms with kinetic energy up to $5 \mathrm{keV}$ were simulated. The initial directions of motion of recoils were chosen randomly. We present results for 2 events at each energy.

The average number of Frenkel pairs produced per cascade is $5.5,11$ and 27 for 1,2 and 5 $\mathrm{keV}$ events, respectively. They correspond to a defect production efficiency of 55,55 and $54 \%$ of the prediction of binary collision models [26] where defect production is described as $N_{N R T}=0.8 \mathrm{Ep} / 2 \bar{E}_{\mathrm{d}}$. Here, $\bar{E}_{\mathrm{d}}$ is the effective average threshold energy for Frenkel pair production. The value of $\bar{E}_{d}$ employed in the present study is $40 \mathrm{eV}$ which is suggested in the 
ASTM standard [24]. The defect production efficiencies obtained here for bcc vanadium are somewhat higher than those of other fcc metals in this energy range. Although these results are for limited statistics, the trend is in agreement with the previous work of Bacon et al. [6-8], which shows that the efficiency of bcc iron is higher than those of other close-packed metals. Furthermore, the defect clustering efficiencies obtained here for vanadium are similar to those of bcc iron [6,7], as shown in Fig. 4; a higher fraction of produced defects is single- and di-defects and smaller fraction is taken up in clusters for bcc vanadium than those in other fcc metals [3-6]. This suggests that direct cluster formation in cascades is greatly influenced by lattice type, and seems to be consistent with experimental observation of lower probability of cascade collapse in bcc metals than that in fcc [27].

\section{SUMMARY}

We modified the EAM interatomic potential for vanadium of Johnson and $\mathrm{Oh}$ to describe high energy part of atom interaction by connecting it with the universal ZBL-potential, and investigated the displacement threshold energies and melting temperature of the system. We further used this potential to investigate defect production during irradiation in vanadium. Our cascade simulation results show a higher production efficiency of defects and lower defect clustering in bcc vanadium compared to other fcc metals. This is similar to results in bcc iron, suggesting that defect production in cascades, including clustering features, are strongly dependent on lattice type.

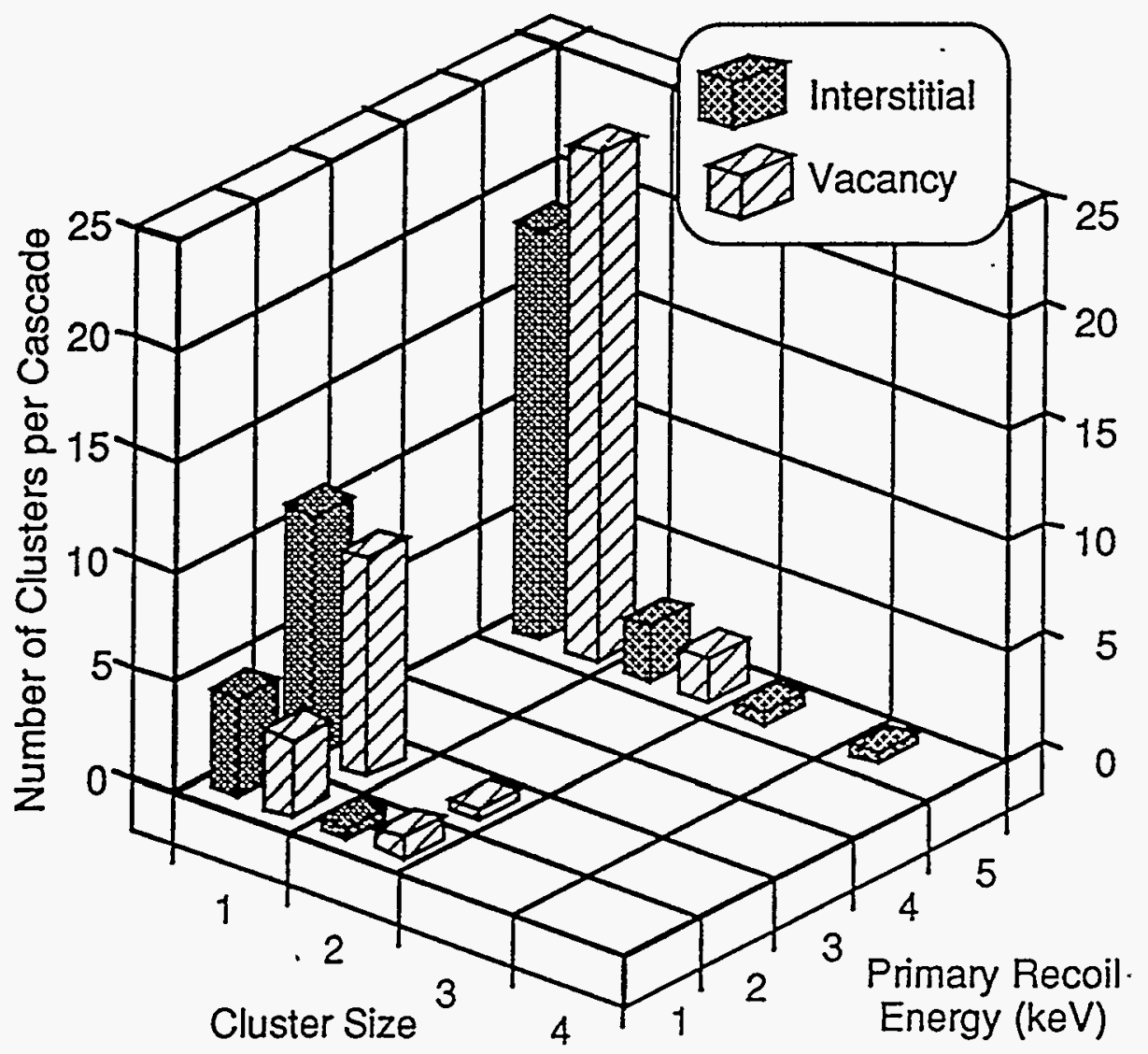

Fig. 4 Size distribution of cascade defects in vanadium at $10 \mathrm{~K}$ as a function of recoil energy. 


\section{ACKNOWLEDGMENTS}

This work was performed under the auspices of the U.S. Department of Energy by Lawrence Livermore National Laboratory under Contract No. W-7405-Eng-48, and supported by a Grant-in-Aid from the Ministry of Education, Science and Culture of Japan. One of the authors (K.M.) wishes to acknowledge the JSPS (Japan Society for the Promotion of Science) Research Fellowships for Young Scientists.

\section{REFERENCES}

1. D.N. Seidman, R.S. Averback and R. Benedek, Phys. Status Solidi (b) 144, 85 (1987).

2. C.H. Woo and B.N. Singh, Phys. Status Solidi (b) 159, 609 (1990); J. Nucl. Mater. 179181, 1207 (1991).

3. T. Diaz de la Rubia, R.S. Averback, R. Benedek and I.M. Robertson, Rad. Eff. Def. Sol. 113, 39 (1990).

4. T. Diaz de la Rubia and M.W. Guinan, Mater. Sci. Forum 97-99, 23 (1992).

5. A.J.E. Foreman, W.J. Phythian and C.A. English, Philos. Mag. A 66, 671 (1992).

6. W.J. Phythian, R.E. Stoller, A.J.E. Foreman, A.F. Calder and D.J. Bacon, J. Nucl. Mater. 223, 245 (1995).

7. D.J. Bacon, A.F. Calder, F. Gao, V.G. Kapinos and S.J. Wooding, Nucl. Instr. and Meth. B 102, 37 (1995).

8. A.F. Calder and D.J. Bacon, J. Nucl. Mater. 207, 25 (1993).

9. T. Diaz de la Rubia and M.W. Guinan, J. Nucl. Mater. 174, 151 (1990).

10. J.B. Adams and S.M. Foiles, Phys. Rev. B 41, 3316 (1990).

11. R.A. Johnson and D.J. Oh, J. Mater. Res. 4, 1195 (1989).

12. M.W. Finnis and J.E. Sinclair, Philos. Mag. A 50, 45 (1984); 53, 161 (E) (1986).

13. R. Rebonato, D.O. Welch, R.D. Hatcher and J.C. Bilello, Philos. Mag. A 55, 655 (1987).

14. Y. Ouyang and B. Zhang, Phys. Lett. A 192, 79 (1994).

15. Y.R. Wang and D.B. Boercker, J. Appl. Phys. 78, 1 (1995).

16. Landorf-Bornstein New Series 25, 115 (1991).

17. P.E.A. Turchi (private communication).

18. W.D. Wilson, L.G. Haggmark and J.P. Biersack, Phys. Rev. B 15, 2458 (1977).

19. S. Proennecke, A. Caro, M. Victoria, T. Diaz de la Rubia and M.W. Guinan, J. Mater. Res. 6, 483 (1991).

20. P. Jung, in Atomic Collisions in Solids, vol. 1, edited. by S. Datz, R. Appleton and C.D. Moak (Plenum, New York, 1975), p.87.

21. A.J.E. Foreman, C.A. English and W.J. Phythian, Philos. Mag. A 66, 655 (1992).

22. F. Gao and D.J. Bacon, Philos. Mag. A 71, 43 (1995).

23. P. Jung, Radiation Effects 35, 155 (1978); J. Nucl. Mater. 117, 70 (1983).

24. ASTM E521, Standard Practice for Neutron Radiation Damage Simulation by ChargedParticle Irradiation, Annual Book of ASTM Standards, vol. 12.02 (American Society of Testing and Materials, Philadelphia, 1994).

25. See, e.g., A Scientific Chronological Table (MARUZEN, Tokyo, 1994) (in Japanese).

26. M.J. Norgett, M.T. Robinson and I.M. Torrens, Nucl. Eng. Des. 33, 50 (1975).

27. C.A. English and M.L. Jenkins, Mater. Sci. Forum 15-18, 1008 (1987). 
Technical information Department - Lawrence Live rmore Nat inal Laboratory Unive rsity of Califomia. - Live more, Califomia 945.51

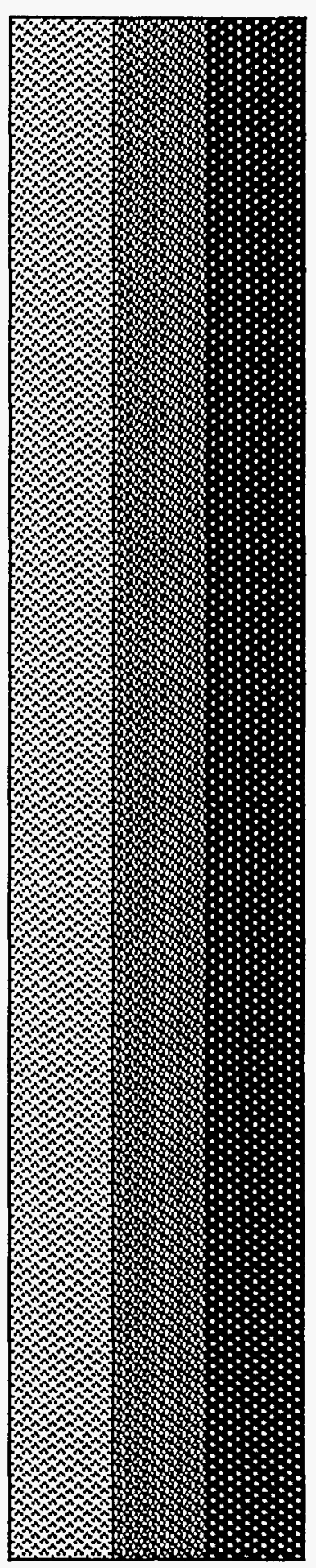

